\title{
Ophiopogon japonicas (Linn. f.) Ker-Gawl. extract ameliorates chronic heart failure in rats
}

\author{
Hu-zhi Cai ${ }^{1}$, Yan-ping Tang ${ }^{2}$, Xin-yu Chen ${ }^{1}$, Hai-bo Xie ${ }^{1}$, Qing-yang Chen ${ }^{1}$, Ze-lin \\ $\mathrm{Xu} \mathbf{u}^{1 *}$ \\ ${ }^{1}$ The First Affiliated Hospital of Hunan University of Traditional Chinese Medicine, Changsha 410007, ${ }^{2}$ Hunan University of \\ Traditional Chinese Medicine, Changsha 410208, Hunan Province, China
}

*For correspondence: Email: xuzelin133@163.com; Tel: +86-731-85600580

\begin{abstract}
Purpose: To investigate the effect of Ophiopogon japonicas (Linn. f.) Ker-Gawl. extract (OJKE) on oxidative stress and hemodynamics in chronic congestive heart failure (CHF) rats.

Methods: The rats were modelled to congestive heart failure (except normal group), and then randomly divided into normal control group, model (untreated) group, captopril group, high-dose, middle-dose and low-dose of OJKE groups. They were treated for 4 weeks as appropriate for each group. At the end of treatment, the hemodynamic function, whole heart weight index, and blood creatinine kinase (CK), as well as superoxide dismutase (SOD), malondialdehyde (MDA), nitric oxide (NO), nitricoxide synthase (NOS) were determined.

Results: Compared with the normal control group, arterial systolic pressure (SBP), diastolic pressure $(D B P)$, mean arterial pressure (MAP), heart rate (HR), left ventricular systolic peak (LVSP), and left ventricular pressure change rate (dp/dt max) significantly decreased $(p<0.05)$, while left ventricular end diastolic pressure (LVEDP), whole heart weight index, blood CK, MDA, NO, NOS significantly increased in the untreated group $(p<0.05)$. A high dose of OJKE significantly improved hemodynamic function, lowered MDA (8.33 $\pm 2.12 \mathrm{nmol} / \mathrm{mL})$ and NO $(20.58 \pm 3.53$ umol/L) levels $(p<0.05)$, and also decreased $C K(0.53 \pm 0.37 \mathrm{U} / \mathrm{mL})$ and NOS $(22.46 \pm 3.29 \mathrm{U} / \mathrm{mL})$ in CHF rats $(p<0.05)$.

Conclusion: OJKE improved adriamycin-induced chronic congestive heart failure in rats significantly.
\end{abstract}

Keywords: Ophiopogon japonicas, Chronic heart failure, Hemodynamic function, Oxidative stress

This is an Open Access article that uses a fund-ing model which does not charge readers or their institutions for access and distributed under the terms of the Creative Commons Attribution License (http://creativecommons.org/licenses/by/4.0) and the Budapest Open Access Initiative (http://www.budapestopenaccessinitiative.org/read), which permit unrestricted use, distribution, and reproduction in any medium, provided the original work is properly credited.

Tropical Journal of Pharmaceutical Research is indexed by Science Citation Index (SciSearch), Scopus, International Pharmaceutical Abstract, Chemical Abstracts, Embase, Index Copernicus, EBSCO, African Index Medicus, JournalSeek, Journal Citation Reports/Science Edition, Directory of Open Access Journals (DOAJ), African Journal Online, Bioline International, Open-J-Gate and Pharmacy Abstracts

\section{INTRODUCTION}

Chronic heart failure (CHF) is a common and complex clinical syndrome that arises from structural or functional cardiac disorder, including changes in electrophysiology, contraction, and energy metabolism [1]. Heart failure (HF) is becoming an increasing disease with an incidence approaching 1 percent of the population over 65 years of age in developed countries [2]. In China, it was reported that the prevalence of $\mathrm{HF}$ in the adult population from ten provinces was $0.9 \%$ [3]. The prognosis for CHF is poor and there are few therapeutic options, heart failure is even worse than many types of cancer [4]. Furthermore, there has been an 
increased medical burden, and HF is becoming a global public health problem. The most effective and commonly used drugs for treatment of HF are angiotensin-converting enzyme (ACE) inhibitors, $\beta$-adrenoceptor blockers, and digitalis [5-7]. The American Heart Association (AHA) and European Society of Cardiology (ESC) have issued and updated the guidelines for diagnosis and management of $\mathrm{CHF}$ [2]. However, $\mathrm{HF}$ is still a leading cause of death worldwide [8].

Therefore, it is necessary to seek novel effective drugs for HF. Traditional Chinese Medicine has significant advantage in the treatment of complex multifactor diseases by targeting multiple pathways to improve therapeutic efficacy and could reduce drug-related side effects and drug resistance. TCMs such as Shengmai [9], Sini decoction [10], Shuanglong formula [11], and Huangqi injection [12] have obviuos therapeutic effects in the treatment of cardiovascular diseases.

It is reported that Ophiopogon japonicas (Linn. f.) Ker-Gawl. extract (OJKE) promoted the blood circulation and removed blood stasis, tonified the blood and arresting bleeding, and alleviated pain $[13,14]$. However, the effect of OJKE on the cardiovascular diseases has not been reported yet. In this experiment, the effect of OJKE on chronic congestive heart failure (CHF) was studied in the adriamycin-induced CHF rats.

\section{EXPERIMENTAL}

\section{Preparation of OJKE}

The plant material Ophiopogon japonicas (Linn. f.) Ker-Gawl. were collected from Dali City, Yunnan Province in China in May 2017. Taxonomic identification of the plant was performed by Professor Qiang Zhang of Hunan University of Traditional Chinese Medicine, in China. A voucher specimen (no. OJKE 20170523) was deposited in College of Pharmacy, Hunan University of Traditional Chinese Medicine, China for future reference.

The herbal samples Ophiopogon japonicas (Linn. f.) Ker-Gawl. was dried in an oven. OJKE was obtained by steeping the dried Ophiopogon japonicas (Linn. f.) Ker-Gawl. in water at $60^{\circ} \mathrm{C}$ three times, each for one hour before first drying in an oven and then freeze-drying. At last, the OJKE was obtained. The yield was $62.5 \%$.

\section{Animals}

SD male rats, weighing $(180 \pm 20) \mathrm{g}$, were purchased from the Experimental Animal Center of Hunan Province (Certificate no. SYXK 20120003). Each rat was raised in single cage, at the environment temperature of $20 \pm 2{ }^{\circ} \mathrm{C}$, humidity 55 - $65 \%$. They were bred with forage for rodents and were free access to water. The rat experiment was approved by the Animal Care and Use Committee of The First Affiliated Hospital of Hunan University of Traditional Chinese Medicine (approval ref no. 20050945) and was carried out in compliance with Directive $2010 / 63 / E U$ on the handling of animals used for scientific purposes [15].

\section{Preparation of chronic heart failure rats and treatment}

Sixty SD rats were divided into normal control group, model group, captopril group, high-dose, middle-dose and low-dose OJKE groups. There were 10 rats in each group. The normal control group rats were treated with intraperitoneal injection of $0.2 \mathrm{~mL}$ saline. Other rats were administrated by intraperitoneal injection of adriamycin hydrochloride $(2 \mathrm{mg} / \mathrm{kg})$ once a week. And it was repeated for 6 times. Six weeks later, two rats were randomly selected from the survived rats with heart failure for detection of the cardiac function, and the unsuccessful modeling rats were continuously modeled for four weeks. The administration of drugs was given from the 7th week of the modeling.

The rats in the normal control group and the model group were intragastrically administrated with $2 \mathrm{~mL}$ saline, once a day; the rats in the OJKE group were administrated intragastrically of OJKE. The dosages were $2.5,5.0$ or $10.0 \mathrm{~g} / \mathrm{kg}$ once a day respectively. The rats in the captopril group were administrated of captopril (100 $\mathrm{mg} / \mathrm{kg}$ ) once each day. The administration lasted for four weeks.

\section{Determination of cardiac function and hemodynamics}

Twenty-four hours after the last intragastrically administration, the rats were anesthetized by intraperitoneal injection of $20 \%$ urethane in 6 $\mathrm{mL} / \mathrm{kg}$, and were fixed on a table in a spinal position. The right common carotid artery was separated with a ventricular canula (cardiac catheter I $\mathrm{mm}$ in diOJKEter), which was connected with Biopac multichannel physiologic sign collection and processing system via a pressure transducer the SBP, DBP, MAP, HR were recorded. Then the cardiac catheter was slowly pushed, and at the same time the pressure oscilloscope was observed. When the wave form was changed and the pulse pressure was increased, it indicates the cardiac catheter 
enters into the left ventricle. After stabilization for $3 \mathrm{~min}$, the LVSP, LVEDP, + $\mathrm{dp} / \mathrm{dt}_{\max }$, and $\mathrm{dp} / \mathrm{dt}_{\max }$ were recorded. For the hemodynamic indices, 5 sections were respectively taken for calculation of the mean value. Then, $10 \mathrm{~mL}$ blood was taken from the abdominal aorta, $5 \mathrm{~mL}$ added with $200 \mathrm{uL}$ EDTANa $\mathrm{Na}_{2}$ and $5 \mathrm{~mL}$ with no anticoagulant, which were centrifuged at $3000 \mathrm{rpm}$ for $15 \mathrm{~min}$. The plasma and serum were kept for other index detection.

\section{Determination of heart weight index}

After hemodynamic detection and the blood sampling, the heart was rapidly separated and the water was absorbed with a filter paper. The heart was weighted, and the heart weight index was calculated. The whole heart weight index = heart weight $(\mathrm{mg})$ / body weight $(\mathrm{g})$.

\section{Blood biochemical test}

CK, MDA, SOD, NO and NOS in the plasma and serum were determined according to the instruction of the kits (Shenzhen Xin Bo Sheng Biological Technology Co., Ltd., Shenzhen, China).

\section{Statistical analysis}

The results were analyzed using Statistical Package SPSS 16.0 (SPSS Inc, Chicago, USA). The data are expressed as mean \pm standard error of mean (SEM) and analyzed by one-way analysis of variance (ANOVA) followed by Dunnett's t-test. A value of $p<0.05$ was considered statistically significant.

\section{RESULTS}

\section{Effect of OJKE on heart weight index}

Heart weight index (HWI) in the model group was higher than that in the normal control group $(p<$ 0.05 ), indicating that there were myocardial hypertrophy or stasis of blood in the model group. Compared with model group, HWI was not significantly changed in captopril and OJKE treatment groups (Table 1).

Table 1: Heart weight index (HWI) for CHF rats $(n=$ 10)

\begin{tabular}{lcc}
\hline Group & Dose $\mathbf{( g / k g )}$ & HWI $(\mathbf{m g} / \mathbf{g})$ \\
\hline Normal control & - & $1.86 \pm 0.12$ \\
Untreated & - & $2.89 \pm 0.13$ \\
Captopril & 0.10 & $2.26 \pm 0.10$ \\
OJKE-L & 2.5 & $2.64 \pm 0.19$ \\
OJKE-M & 5.0 & $2.53 \pm 0.14$ \\
OJKE-H & 10.0 & $2.49 \pm 0.19$
\end{tabular}

Compared with model group, $p<0.05, " p<0.01$. OJKE-L: low dose of OJKE, OJKE-M: medium dose of OJKE, OJKE-H: high dose of OJKE

\section{Effect of OJKE on hemodynamic index}

Compared with the normal control group, SBP, DBP, MAP, HR, LVSP, dp/dt $\mathrm{d}_{\max }$ were significantly decreased $(p<0.05)$, and LVEDP was significantly increased in the model group $(p<$ 0.01). OJKE can significantly improve vasomotoricity and the left ventricular function of the rats with $\mathrm{CHF}(p<0.05)$; while captopril did not have significant effects on vasomotoricity and the left ventricular function of the CHF rats (Table 2 and Table 3).

Table 2: Hemodynamic index for CHF rats $(n=10)$

\begin{tabular}{lccccc}
\hline Group & Dose $\mathbf{( g / k g )}$ & SBP $(\mathbf{m m H g})$ & DBP $(\mathbf{m m H g})$ & MAP $(\mathbf{m m H g})$ & HR (beats/min) \\
\hline Normal control & - & $128.36 \pm 10.28^{*}$ & $117.38 \pm 13.37$ & $109.33 \pm 16.24$ & $427.28 \pm 29.16^{*}$ \\
Untreated & - & $76.28 \pm 14.17$ & $83.24 \pm 19.24$ & $82.29 \pm 11.27$ & $323.14 \pm 31.28$ \\
Captopril & 0.10 & $115.42 \pm 19.21$ & $91.16 \pm 28.17$ & $98.43 \pm 20.27$ & $394.18 \pm 37.15$ \\
OJKE-L & 2.5 & $98.34 \pm 15.16$ & $81.14 \pm 24.27$ & $85.31 \pm 14.32$ & $368.45 \pm 29.26$ \\
OJKE-M & 5.0 & $100.24 \pm 12.28^{*}$ & $94.37 \pm 16.28$ & $96.18 \pm 10.38$ & $375.28 \pm 27.43$ \\
OJKE-H & 10.0 & $116.27 \pm 10.64^{* *}$ & $117.12 \pm 16.16^{* *}$ & $114.16 \pm 14.37^{* *}$ & $409.34 \pm 34.27^{*}$ \\
\hline
\end{tabular}

Compared with control group, $p<0.05$, " $p<0.01$. OJKE-L: low dose of OJKE, OJKE-M: medium dose of OJKE, OJKE$\mathrm{H}$ : high dose of OJKE

Table 3: More hemodynamic indices for CHF rats $(n=10)$

\begin{tabular}{|c|c|c|c|c|c|}
\hline Group & $\begin{array}{l}\text { Dose } \\
(\mathrm{g} / \mathrm{kg})\end{array}$ & $\begin{array}{c}\text { LVSP } \\
(\mathrm{mmHg})\end{array}$ & $\begin{array}{l}\text { LVEDP } \\
(\mathrm{mmHg})\end{array}$ & $+d_{p} / d_{\max }$ & $-d p / d t_{\max }$ \\
\hline Normal control & - & $164.37 \pm 13.27$ & $9.42 \pm 1.34$ & $3654.26 \pm 424.31$ & $3743.21 \pm 408.28$ \\
\hline Untreated & - & $95.17 \pm 18.26$ & $26.27 \pm 1.64$ & $2348.17 \pm 605.23$ & $2416.38 \pm 607.42$ \\
\hline Captopril & 0.10 & $108.23 \pm 29.22$ & $14.37 \pm 2.06$ & $3218.11 \pm 756.45$ & $3286.14 \pm 708.35$ \\
\hline OJKE-L & 2.5 & $114.08 \pm 16.17$ & $17.86 \pm 1.57$ & $2435.27 \pm 534.16$ & $2415.28 \pm 576.39$ \\
\hline OJKE-M & 5.0 & $128.19 \pm 14.05^{*}$ & $14.26 \pm 2.42$ & $3124.18 \pm 443.27^{\pi}$ & $3142.27 \pm 526.31$ \\
\hline OJKE-H & 10.0 & $137.26 \pm 11.28^{*}$ & $11.26 \pm 2.39^{*}$ & $3348.27 \pm 542.14$ & $3649.34 \pm 517.16$ \\
\hline
\end{tabular}

Compared with control group, $p<0.05, " p<0.01$. OJKE-L: low dose of OJKE, OJKE-M: medium dose of OJKE, OJKE$\mathrm{H}$ : high dose of OJKE 
Table 4: Blood levels of CK, SOD, MDA, NO and NOS of the rats $(n=10)$

\begin{tabular}{lcccccc}
\hline Group & Dose $(\mathbf{g} / \mathbf{k g})$ & $\mathbf{C K}(\mathbf{U} / \mathbf{m L})$ & $\begin{array}{c}\text { SOD } \\
\text { (U/mgprot) }\end{array}$ & $\begin{array}{c}\text { MDA } \\
\text { (nmol/mL) }\end{array}$ & NO (umol/L) & $\begin{array}{c}\text { NOS } \\
(\mathbf{U} / \mathbf{m L})\end{array}$ \\
\hline Normal control & - & $0.28 \pm 0.11$ & $112.14 \pm 6.54$ & $6.53 \pm 1.43$ & $15.36 \pm 1.86$ & $19.27 \pm 3.08$ \\
Untreated & - & $0.87 \pm 0.13$ & $67.83 \pm 4.24$ & $16.27 \pm 2.10$ & $37.14 \pm 3.25$ & $44.26 \pm 3.18$ \\
Captopril & 0.10 & $0.38 \pm 0.11^{\prime \prime}$ & $87.05 \pm 7.84$ & $8.26 \pm 1.42^{\prime \prime}$ & $29.18 \pm 3.12^{\prime \prime}$ & $35.28 \pm 2.43$ \\
OJKE-L & 2.5 & $0.77 \pm 0.24$ & $71.37 \pm 8.23$ & $13.27 \pm 2.16$ & $28.14 \pm 3.27$ & $38.34 \pm 4.15$ \\
OJKE-M & 5.0 & $0.67 \pm 0.21$ & $79.28 \pm 7.39$ & $10.37 \pm 2.18$ & $26.24 \pm 3.17$ & $31.27 \pm 3.85$ \\
OJKE-H & 10.0 & $0.53 \pm 0.37$ & $72.18 \pm 6.42$ & $8.33 \pm 2.12$ & $20.58 \pm 3.53^{\prime \prime}$ & $22.46 \pm 3.29^{\prime \prime}$ \\
\hline
\end{tabular}

Compared with control group, $p<0.05, " p<0.01$. OJKE-L: low dose of OJKE, OJKE-M: medium dose of OJKE, OJKE-H: high dose of OJKE

\section{Effect of OJKE on blood CK, SOD, MDA, NO and NOS}

Compared with the normal control group, CK and NOS activities, MDA and NO levels were significantly increased $(p<0.01)$, and SOD activity was significantly decreased in the model group $(p<0.01)$. Compared with the model group, blood CK activity, MDA and NO levels were significantly decreased in the captopril group ( $p<0.01)$, and MDA and NO levels, NOS activity significantly decreased in the OJKE group $(p<0.01)$ (Table 4$)$.

\section{DISCUSSION}

Hemodynamic parameters are important indices reflecting cardiac functions. OJKE strengthened the diastolic and contractile functions of the artery and left ventricle, and significantly improved the left ventricular function with no change in heart rate, showing better effects than that of captopril.

Oxidative stress is one key factor for heart failure [16]. In heart failure, a large number of active oxygen families and nitric oxide are produced. A lot of free radicals induce lipid peroxidation, injuring the cellular membrane and inducing inflammation and cell apoptosis. In physiologic state, the organism has an anti-oxidation system, for example SOD can clear away superoxide anions in time, and reduce lipid peroxidation. In physiologic state, NO has the function of dilating blood vessels. However, a great quantity of NO could not only induce the production of free radicals, but also mediate serious neurotoxicity and cytotoxicity, promoting injury of tissues. Synthesis of NO needs the participation of NOS [17]. It was found in the experiment that OJKE could significantly decrease the blood MDA and NO levels, and CK and NOS activities, without the effect on blood SOD activity. Therefore, it is suggested that the improvement of oxidative stress state by OJKE in CHF rats was not carried out by SOD.

\section{CONCLUSION}

This study indicates that OJKE improves chronic congestive heart failure in CHF model rats, and this is possibly related to the alleviation of oxidative stress and improvement in left ventricular function. OJKE has the potential to be developed as an alternative medicine for treating chronic heart failure in future.

\section{DECLARATIONS}

\section{Acknowledgement}

This study was supported by the National Natural Science Fund (nos. 81173213 and 81704061), Scientific Research Project of Hu'nan Provincial Education Department (no. 16C1238), and Funded Project of Hunan Provincial Administration of Traditional Chinese Medicine (no. 201651).

\section{Conflict of interest}

No conflict of interest is associated with this work.

\section{Contribution of authors}

We declare that this work was done by the authors named in this article and all liabilities pertaining to claims relating to the content of this article will be borne by the authors. Ze-lin Xu designed all the experiment and revised the paper. Hu-zhi Cai,

Yan-ping Tang, Xin-yu Chen and Hai-bo Xie performed the experiments, and

Qing-yang Chen wrote the manuscript. Hu-zhi Cai and Yan-ping Tang contributed equally to this work, and they are co-first authors.

\section{Open Access}

This is an Open Access article that uses a funding model which does not charge readers or their institutions for access and distributed under the 
terms of the Creative Commons Attribution License (http://creativecommons.org/licenses/by/ 4.0) and the Budapest Open Access Initiative (http://www.budapestopenaccessinitiative.org/rea d), which permit unrestricted use, distribution, and reproduction in any medium, provided the original work is properly credited.

\section{REFERENCES}

1. Dickstein K, Cohen-Solal A, Filippatos G. Guidelines for the diagnosis and treatment of acute and chronic heart failure. Eur J Heart Fail 2008; 10: 933-989.

2. Hunt $S A$, Abraham WT, Chin MH, Feldman AM. ACC/AHA 2005 guideline update for the diagnosis and management of chronic heart failure in the adult a report of the OJKErican College of Cardiology/OJKErican Heart Association Task Force on Practice Guidelines. Circulation 2005; 112: e154-e235.

3. Gu D, Huang G, He J. Investigation of prevalence and distributing feature of chronic heart failure in Chinese adult population. Chin J Cardiol 2002; 31: 3-6.

4. McMurray J, Stewart S. The burden of heart failure. Eur Heart J 2002; 4: 50-58.

5. Costanzo MR, Guglin ME, Saltzberg MT. Ultrafiltration versus intravenous diuretics for patients hospitalized for acute decompensated heart failure. J Am Coll Cardiol 2007; 49: 675-683.

6. Yusuf $S$, Sleight $P$, Pogue J. Effects of an angiotensinconverting-enzyme inhibitor, ramipril, on cardiovascular events in high-risk patients. The Heart Outcomes Prevention Evaluation Study Investigators. N Engl $J$ Med 2000; 342: 145-153.

7. López-Sendó J, Swedberg K, McMurray J. Expert consensus document on $\beta$-adrenergic receptor blockers The Task Force on Beta-Blockers of the European Society of Cardiology. Eur. Heart J 2004; 25: 1341 1362.
8. Torabi A, Cleland JG, Khan NK. The timing of development and subsequent clinical course of heart failure after a myocardial infarction. Eur. Heart J. 2008; 29: 859-870.

9. Chen J, Yao Y, Chen H. Shengmai (a traditional Chinese herbal medicine) for heart failure. Cochrane Database Syst Rev 2012; 11: 41-43.

10. Tan G, Liao W, Dong X, Yang G. Metabonomic profiles delineate the effect of traditional Chinese medicine Sini decoction on myocardial infarction in rats. PLOS One 2012; 7: e34157.

11. Liang $X$, Chen $X$, Liang $Q$, Zhang $H$. Metabonomic study of Chinese medicine Shuanglong formula as an effective treatment for myocardial infarction in rats. $J$ Proteome Res 2010; 10: 790-799.

12. Fu S, Zhang J, Menniti-Ippolito F, Gao X. Huangqi injection (a traditional Chinese patent medicine) for chronic heart failure: A systematic review. PLOS One 2011; 6: e19604.

13. Yong Chen. Clinical observation on the treatment of 50 cases of chronic heart failure with modified Zhenwu Decoction. Guangming Tradit Chin Med 2006; 21: 3436.

14. Zhu BB, Guo W, Huang L, Yang J. Effect of Zhenwu Decoction on ET and CGRP levels in chronic congestive heart failure model rats. Hubei Tradit Chin Med 2005; 26: 49-51.

15. European Commission [homepage on the internet]. Directive 2010/63/EU on the protection of animals used for scientific purposes [cited 2013 Jan 16]. Available from:

http://ec.europa.eu/environment/chemicals/lab_animals/l egislation_en.htm.

16. Seddon $M$, Looi $Y H$, Shah $A M$. Oxidative stress and redox signalling in cardiac hypertrophy and heart failure. Heart 2006; 12: 131-138.

17. Azevedo PS, Minicucci MF, Santos PP. Energy metabolism in cardiac remodeling and heart failure. Cardiol Rev 2013; 21: 135-140. 